\title{
Compositional mapping of surfaces in atomic force microscopy by excitation of the second normal mode of the microcantilever
}

\author{
Tomás R. Rodríguez and Ricardo García ${ }^{a}$ \\ Instituto de Microelectrónica de Madrid, CSIC, Isaac Newton 8, 28760 Tres Cantos, Madrid, Spain
}

(Received 4 August 2003; accepted 20 November 2003)

\begin{abstract}
We propose a method for mapping the composition of a surface by using an amplitude modulation atomic force microscope operated without tip-surface mechanical contact. The method consists in exciting the first two modes of the microcantilever. The nonlinear dynamics of the tip motion, the coupling of its first two modes, and the sensitivity of the second mode to long-range attractive forces allows us to use this mode to probe compositional changes while the signal from the first mode is used to image the sample surface. We demonstrate that the second mode has a sensitivity to surface force variations below $10^{-11}$ N. (C) 2004 American Institute of Physics.
\end{abstract}

[DOI: $10.1063 / 1.1642273$ ]

Combined mapping of topography and chemical composition of biological, organic, and heterogeneous surfaces is one of the goals of microscopic techniques. Over the past 10 years amplitude modulation atomic force microscopy (AMAFM) has been very successful for obtaining high-resolution images of a large variety of materials and molecules such as DNA, membrane proteins, polymer surfaces, silicon dots, or inorganic crystals. ${ }^{1}$ In AM-AFM a nanometer-size probe is excited at or near its free resonance frequency. The surface is imaged while the feedback electronics adjust the tip-surface separation to keep a constant amplitude. The phase shift existing between the excitation and the cantilever's response has been proposed and applied to generate compositional maps of heterogeneous surfaces. ${ }^{2-8}$ However, phase images do not reveal changes in conservative tip-surface interactions because phase shifts obtained at constant amplitude are directly related to energy dissipation processes. ${ }^{9-11}$ Furthermore, in those cases phase shift images imply tip-sample mechanical contact, which for some single biological molecules involve the irreversible deformation of the sample. ${ }^{12}$

The current interpretation of AM-AFM is based on point-mass models, i.e., the microcantilever-tip system is considered to have a single resonance frequency. ${ }^{13-16}$ This approximation has been successful to describe the complex nonlinear dynamics of the tip motion. However, point-mass models ignore higher oscillation modes of the cantilever, which in turn has undermined the potential of AM-AFM for obtaining compositional contrast through elastic interactions.

A description of the cantilever as a continuous beam has been rarely applied in AFM. The thermal noise of a rectangular cantilever has been calculated in air ${ }^{17}$ and liquids. ${ }^{18,19}$ Stark and Heckel have calculated the contribution of the higher harmonics to the tip deflection under the influence of a periodic external repulsive force. ${ }^{20}$ They have also suggested the use of the third mode of V-shaped cantilevers to record phase images. ${ }^{21}$ Higher harmonics have also been suggested to measure Young modulus when the tip interacts under strong repulsive forces. ${ }^{22-24}$

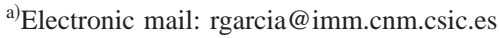

In this letter we study the tip motion of a rectangular microcantilever in the proximity of a surface. The microcantilever has a hemispherical tip attached at its free end. The theoretical simulations led us to propose a method for mapping simultaneously the topography and the chemical composition of a sample surface in noncontact AM-AFM. The method consists of exciting the first two modes of the microcantilever. The output signal of the first mode is used to image the topography of the sample while the second mode is used to map changes in the composition of the atoms or molecules under the tip. The simulations were performed by modeling the three dimensional microcantilever as a rectangular beam and applying the Newton equation. ${ }^{25}$ Then the dynamic deflection function $w(x, t)$ is described by

$$
\begin{aligned}
\frac{E I}{L^{4}} & \frac{\partial^{4}}{\partial x^{4}}\left[w(x, t)+a_{1} \frac{\partial w(x, t)}{\partial t}\right]+\rho b h \frac{\partial^{2} w(x, t)}{\partial t^{2}} \\
& =F_{\mathrm{exc}}(x, t)+F_{\mathrm{med}}(x, t)+F_{t-s}(x, t),
\end{aligned}
$$

where $x$ is the normalized coordinate along the beam, $E$ is the Young modulus, $I$ is the moment of inertia, $\rho$ is the mass density, and $a_{1}$ is the internal damping of the cantilever, while $L, b$, and $h$ are, respectively, the length, width, and height of the cantilever. The boundary conditions assume one end of the cantilever clamped $(x=0)$ and the other free $(x$ $=1) . F_{\text {exc }}, F_{\text {med }}$, and $F_{t-s}$ are the excitation, hydrodynamic damping with the medium and interaction forces per unit of length, respectively. The excitation force includes first and second mode components

$$
F_{\text {exc }}(x, t)=\frac{\delta(1-x)}{L}\left(F_{1} \cos \omega_{0,1} t+F_{2} \cos \omega_{0,2} t\right),
$$

where $\omega_{0,1}$ and $\omega_{0,2}$ are the frequencies of the first and second normal modes of the free cantilever. The excitation force and the tip-surface force are applied at the tip position. Longand short-range interactions forces are included in the model following the approximations given in Ref. 13. In particular, van der Waals forces are calculated by the expression

$$
F_{\mathrm{vdw}}=-\frac{H R}{6 d^{2}}
$$




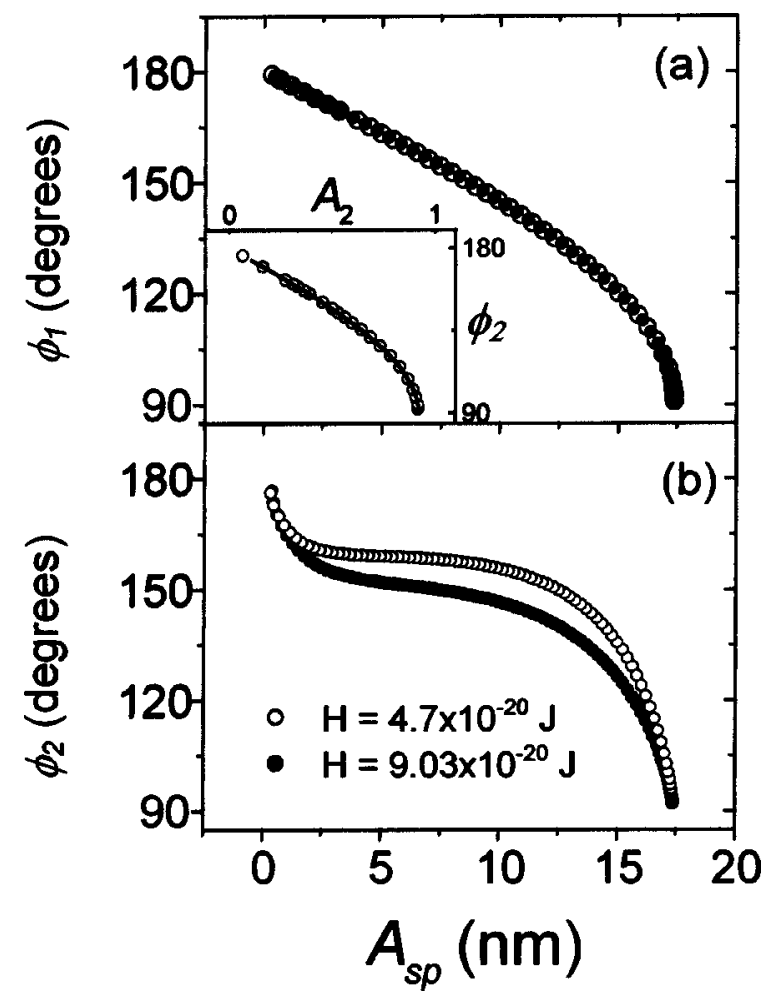

FIG. 1. Phase shift dependence on set point amplitude for two different Hamaker values. (a) First mode phase shift $\phi_{1}$. The inset shows the dependence of $\phi_{2}$ on its own amplitude. In both cases the phase shift is independent on $H$ values. (b) Second mode phase shift as a function of $A_{\text {sp }}$ and $H$ values. Full and empty circles corresponds to $H=9.03 \times 10^{-20} \mathrm{~J}$ and 4.7 $\times 10^{-20} \mathrm{~J}$, respectively.

where $H, R$, and $d$ are the Hamaker constant, tip's radius, and tip-surface instantaneous distance. To solve Eq. (1), the deflection is expressed in a base of the normal modes of the free cantilever. The boundary conditions imply that the deflection can be separated into spatial and temporal components. Then the initial equation is equivalent to a system of coupled anharmonic oscillators, one equation for each mode. We have modeled a cantilever with $L, b, h, E, R$, and $\rho$ of $225,40,1.8 \mu \mathrm{m}, 170 \mathrm{GPa}, 20 \mathrm{~nm}$, and $2320 \mathrm{~kg} / \mathrm{m}^{3}$, respectively,

$$
F_{\text {med }}(x, t)=-a_{0} \partial w(x, t) / \partial t
$$

is set to obtain amplitude versus frequency curves for the first two modes that are fitted to lorentzians with $f_{0,1}, k_{1}$, $A_{01}$, and $Q_{1}$ of $20 \mathrm{~nm}, 48.9 \mathrm{kHz}, 0.9 \mathrm{~N} / \mathrm{m}, 17.4 \mathrm{~nm}$, and 255 for the first mode and $f_{0,2}, k_{2}, A_{02}$, and $Q_{2}$ of $306.6 \mathrm{kHz}$, $35.22 \mathrm{~N} / \mathrm{m}, 0.92 \mathrm{~nm}$, and 1002.4 for the second mode $\left(a_{0}\right.$ $=2 \times 10^{-4} \mathrm{~kg} / \mathrm{m} \mathrm{s}$ and $\left.a_{1}=10^{-10} \mathrm{~s}\right)$. Those values represent some of the common cases for tapping-mode cantilevers. We numerically solve the system of equations for the first three modes $(48.9,306.6$, and $858.2 \mathrm{kHz}$, respectively) with three coupled fourth-order Runge-Kutta algorithms.

The dependence of the phase shift of the first and second mode $\left(\phi_{1}\right.$ and $\left.\phi_{2}\right)$ with respect to the set point amplitude $\left(A_{\mathrm{sp}}\right)$ are shown in Fig. 1. The simulations describe two different interfaces, $\mathrm{SiO}_{2}$-air-water-mica (water for short) as a representative of many experimental situations where a very thin film of water is absorbed on the mica and $\mathrm{SiO}_{2}$-air-mica (mica) that are parametrized by Hamaker Downloaded 12 Mar 2010 to 161.111.235.252. Redistribution subject

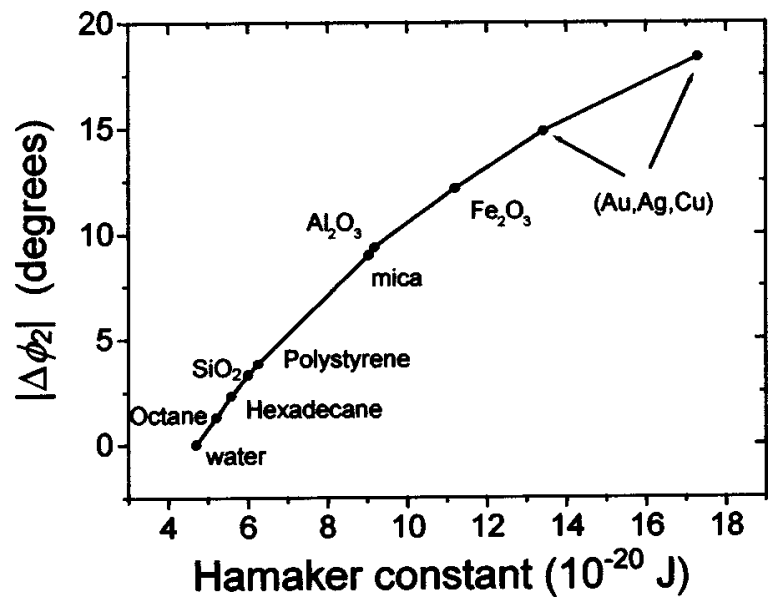

FIG. 2. Phase shift difference $\left|\Delta \phi_{2}\right|=\left|\phi_{2}(H)-\phi_{2}\left(\mathrm{H}_{2} \mathrm{O} / \mathrm{mica}\right)\right|$ for some representative materials. Notice that the simulated range covers almost all the available range of $H$ values of the condensed materials.

constants of $4.7 \times 10^{-20}$ and $9.03 \times 10^{-20} \mathrm{~J}$, respectively. ${ }^{26}$ We have verified that in all the cases studied here the lower end of oscillation never experiences repulsive forces.

The independence of $\phi_{1}$ on Hamaker values is a consequence of $A_{1} \gg A_{2}$. The free amplitude of the first mode is $A_{1} \approx 17 \mathrm{~nm}$ while the free amplitude of the second mode is $A_{2} \approx 0.9 \mathrm{~nm}$. The earlier leads to $A_{\mathrm{sp}} \approx A_{1}$ although excitation forces are comparable, $F_{1}$ and $F_{2}$ are 60 and $20 \mathrm{pN}$, respectively. This implies that the first mode behaves as a single anharmonic oscillator. It also implies that standard AMAFM topographic imaging is unaltered by the excitation of higher modes. The inset shows that $\phi_{2}$ vs $A_{2}$ is also insensitive to changes in the strength of the attractive force which reflects that the second mode also behaves as a single anharmonic oscillator with respect to the relationship between its own amplitude and phase. On the other hand, $\phi_{2}$ shows a strong dependence on Hamaker values when it is plotted versus $A_{\mathrm{sp}}$. A phase shift difference of $9.0^{\circ}$ is obtained for the mica and water-mica interfaces.

Figure 2 shows the phase shift difference $\left|\Delta \phi_{2}\right|$ $=\mid \phi_{2}(H)-\phi_{2}\left(\mathrm{H}_{2} \mathrm{O} /\right.$ mica $) \mid$ for some representative tip-airsample interfaces at a constant amplitude $\left(A_{\mathrm{sp}}=10 \mathrm{~nm}\right)$. The curve shows that $\left|\Delta \phi_{2}\right|$ grows monotonously with $H$ increasing from $\mathrm{H}_{2} \mathrm{O}$ to metal surfaces. More interestingly it distinguishes between similar organic samples, for example a $\Delta \phi_{2}=1^{\circ}$ is obtained between octane and hexadecane. Furthermore, a $5 \%$ change in $H$ (from 9.03 to $8.58 \times 10^{-20} \mathrm{~J}$ ) will give a phase shift difference of $0.7^{\circ}$ well above experimental noise $\left(\sim 0.1^{\circ}\right)$. This implies a sensitivity to changes in the maximum force of $10 \mathrm{pN}$. We remark that changes in the normal force between 10 and $100 \mathrm{pN}$ are needed to obtain true atomic resolution images in calcite surfaces ${ }^{27}$ or in reconstructed silicon surfaces. ${ }^{28}$

In general, $\phi_{1}, \phi_{2}, A_{1}$, and $A_{2}$ depend on $H$ values, however, their sensitivity to $H$ variations cancels out when parameters of the same mode are represented because they are not independent. For example, it has been demonstrated that point-mass and continuous models produce very similar results when there is only a single excitation. ${ }^{29}$ Then the phase shift of a given mode can be calculated by generalization of the expression for a single anharmonic oscillator ${ }^{10,11}$ to AIP license or copyright; see http://apl.aip.org/apl/copyright.jsp 


$$
\sin \phi_{i}=\frac{\omega A_{i}}{\omega_{0, i} A_{0 i}}+\frac{Q E_{\mathrm{dis}}^{i}}{\pi k A_{0 i} A_{i}} .
$$

Equation (5) says that at fixed amplitude the phase shift is proportional to the amount of energy dissipated by the tip on the surface which in the absence of inelastic interactions gives a relationship between phase shift and amplitude. However, the dependence on material properties is preserved when crossed representations are used ( $\phi_{i}$ vs $A_{j}$ with $i \neq j$ ). The coupling of the modes induced by the tip-surface interactions and the continuous character of the microcantilever enhances the earlier effect. It allows $\phi_{2}$ to achieve compositional contrast for separations one or two order of magnitude larger than $A_{2}$.

The earlier results have been obtained by assuming van der Waals forces described by Eq. (3). In a realistic experimental setup other forces with different tip-surface dependencies could be present. However, we would like to emphasize that the sensitivity of $\phi_{2}$ to detect force changes is general in the sense that it applies for any nonlinear tipsurface interaction with independence of the specific origin of the force.

Three physical factors are responsible for the observed dependence of the amplitude or phase shift of the second mode on the strength of the tip-surface force, the nonlinear dynamics of the tip motion, the coupling of the normal vibrational modes, and the sensitiveness of the second mode to long-range attractive forces. The simultaneous excitation of the first and second modes of a microcantilever vibrating in the proximity of a surface provides the presence of two coupled nonlinear oscillators. The coupling of the first two modes and the enhanced $Q$ factor of the second mode allows this mode to probe tip-surface forces with a sensitivity to changes in the strength of the attractive force of $10^{-11} \mathrm{~N}$. Those values are comparable or smaller than the attractive force exerted between a single atom in a surface and a nanometer-size tip a few angstroms apart. This demonstrates the feasibility to develop a spatially resolved force spectroscopy with atomic or nanometer resolution for operation in liquids or air.
The authors acknowledge helpful discussions with A. San Paulo. This work was financially supported by the Dirección General de Investigación Científica y Técnica of Spain (PB98-0471) and the European Commission (MONA-LISA, G5RD-CT-2000-00349).

${ }^{1}$ R. García and R. Pérez, Surf. Sci. Rep. 47, 197 (2002), and references therein.

${ }^{2}$ G. Bar, Y. Thomann, and M.-H. Whangbo, Langmuir 14, 1219 (1998).

${ }^{3}$ R. García, J. Tamayo, M. Calleja, and F. García, Appl. Phys. A: Mater. Sci. Process. A66, S309 (1998).

${ }^{4}$ O. P. Behrend, L. Odoni, J. L. Loubet, and N. A. Burnham, Appl. Phys. Lett. 75, 2551 (1999).

${ }^{5}$ G. Reiter, G. Castelein, J. U. Sommer, A. Röttele, and T. Thurn-Albrecht, Phys. Rev. Lett. 87, 226101 (2001).

${ }^{6}$ M. Stark, C. Möller, D. J. Muller, and R. Guckenberger, Biophys. J. 80, 3009 (2001).

${ }^{7}$ M. S. Marcus, R. W. Carpick, D. Sasaki, and M. A. Eriksson, Phys. Rev. Lett. 88, 226103 (2002)

${ }^{8}$ W. W. Scott and B. Bhushan, Ultramicroscopy 97, 151 (2003).

${ }^{9}$ J. Tamayo and R. García, Appl. Phys. Lett. 71, 2394 (1997).

${ }^{10}$ J. Tamayo and R. García, Appl. Phys. Lett. 71, 2394 (1998).

${ }^{11}$ J. P. Cleveland, B. Anczykowski, A. E. Schmid, and V. Elings, Appl. Phys. Lett. 72, 2613 (1998).

${ }^{12}$ A. San Paulo and R. García, Biophys. J. 78, 1599 (2000).

${ }^{13}$ R. García and A. San Paulo, Phys. Rev. B 60, 4961 (1999).

${ }^{14}$ L. Wang, Surf. Sci. 429, 178 (1999).

${ }^{15}$ L. Nony, R. Boisgard, and J. Aimé, J. Chem. Phys. 111, 1615 (1999).

${ }^{16}$ S. I. Lee, S. W. Howell, A. Raman, and R. Reifenberger, Phys. Rev. B 66, 115409 (2002).

${ }^{17}$ H.-J. Butt and M. Jaschke, Nanotechnology 6, 1 (1995).

${ }^{18}$ J. E. Sader, J. Appl. Phys. 84, 64 (1998).

${ }^{19}$ F.-J. Elmer and M. Dreier, J. Appl. Phys. 81, 7709 (1997).

${ }^{20}$ R. W. Stark and W. M. Heckl, Surf. Sci. 457, 219 (2000).

${ }^{21}$ R. W. Stark, T. Drobek, and W. M. Heckl, Appl. Phys. Lett. 74, 3296 (1999).

${ }^{22}$ U. Rabe, J. Turner, and W. Arnold, Appl. Phys. A: Mater. Sci. Process. A66, S277 (1998).

${ }^{23}$ S. C. Minne, S. R. Manalis, A. Atalar, and C. Quate, Appl. Phys. Lett. 68, 1427 (1996).

${ }^{24}$ M. Stark, R. W. Stark, W. Heckl, and R. Guckenberger, Appl. Phys. Lett. 77, 3293 (2000).

${ }^{25}$ L. D. Landau and E. M. Lifshitz, Theory of Eleasticity (Pergamon, Oxford, 1970).

${ }^{26} \mathrm{~J}$. Israelachvili, Intermolecular and Surface Forces (Academic, London, 1992).

${ }^{27}$ F. Ohnesorge and G. Binnig, Science 260, 1451 (1993).

${ }^{28}$ R. Perez, M. C. Payne, I. Stich, and K. Terakura, Phys. Rev. Lett. 78, 678 (1997).

${ }^{29}$ T. R. Rodríguez and R. García, Appl. Phys. Lett. 80, 1646 (2002). 